\title{
Improvements in diffraction efficiency of gratings and microlenses with continuous relief structures
}

\author{
M. Kuittinen ${ }^{1}$, H.P. Herzig, P. Ehbets \\ Inst. of Microtechnology, Univ. of Neuchâtel, Rue A.-L. Breguet 2, CH-2000 Neuchâtel, Switzerland
}

\begin{abstract}
The fabrication of diffractive optical elements by laser-beam writing or gray-tone technology leads to continuous-relief phase elements. The diffaction efficiency of such elements is limited by the resolution of the process. In this paper, we compare the continuous-relief elements with the multilevel elements fabricated by binary technology. In particular, we will show that for similar sampling resolution of the ideal phase function, the continuous profiles have higher efficiencies than the multilevel profiles if the designed phase modulation is $4 \pi$ and more.
\end{abstract}

\section{Introduction}

Nowadays, diffractive optical elements (DOEs) are fabricated routinely by photolithography and subsequent etching in glass or quartz as multilevel surfacerelief structures. These elements can be considered locally as blazed gratings. The diffraction efficiency is determined by the number of phase levels for a phase depth of $2 \pi$. Eight phase levels and more enable higher efficiencies than $94 \%$. However, the number of levels is limited by the resolution of the photolithographic process. In the outer zone of diffractive lenses with a high numerical aperture, or of lenses for short wavelengths, the period of the grating decreases to a few microns. In that case, only two or four phase levels can be fabricated properly by standard lithography. This leads to a poor diffraction efficiency of the elements. Increasing the phase depth to $4 \pi$ or $8 \pi$ does increase the number of phase levels and the local grating pe-

1 On leave from University of Joensuu, Department of Physics, Väisälä Laboratory, P.O. Box 111, FIN-80101 Joensuu, Finland. riod, but it does not increase the diffraction efficiency, because there are still only two phase values per $2 \pi$. We will show that this behaviour is different for continuous relief structures fabricated by gray-tone mask technology [1] or direct laser-writing methods [2].

In section two we investigate the diffraction behavior of continuous grating structures. Starting with multilevel profiles, we approach to the ideal blazed grating by smoothing the profile until the resolution limit. In section three, we discuss the influence of the smoothing on the diffraction efficiency of lenses.

\section{Continuous grating profiles}

Continuous blazed structures have, in an ideal case, $100 \%$ diffraction efficiency. Due to the fabrication limitations these ideal structures can not be realised. In a $N$-step lithographic process, $2^{N}$ equally spaced phase values are used to approximate the ideal blaze within one grating period. This approximation leads to efficiencies higher than $94 \%$ when $N \geq 3$. In the case 


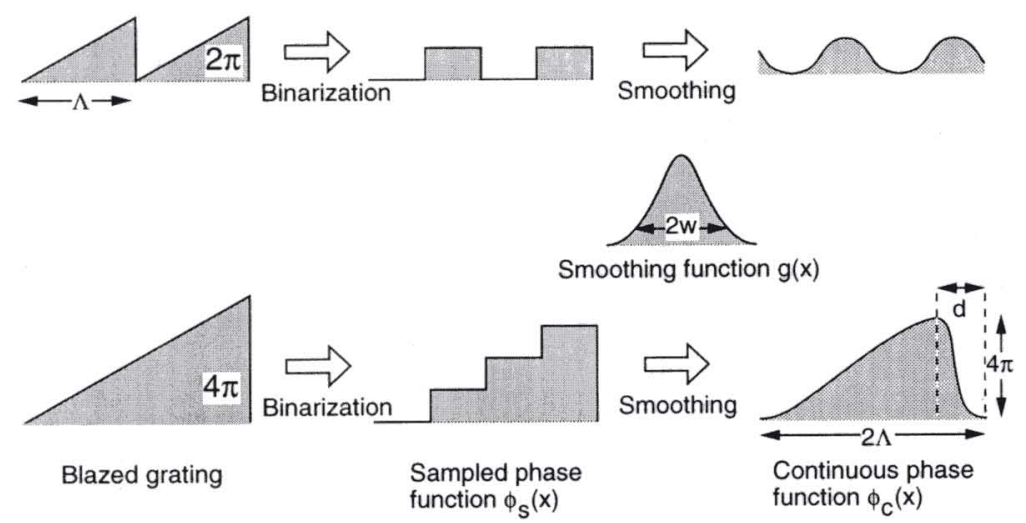

Fig. 1. Convolution of a multilevel grating with a Gaussian signal.

of diffractive lenses with small $F / \#$, the local grating structure on the edge of lenses becomes, however, binary.

This is not much different, if we use a "smooth technology", like gray-tone mask technology or direct laser-beam writing. As long as we work with optical wavelengths, we are limited by diffraction and therefore, we cannot increase the number of resolved pixels. However, if we double the period by increasing the phase depth, suitable smoothing can improve the efficiency of these structures.

We apply a simple model in order to show the principle. In any case, the ideal phase function $\phi_{\mathrm{c}}(x)$ of a continuous grating profile can be modelled by a convolution of a sampled profile $\phi_{\mathrm{s}}(x)$ and smoothing function $g(x)$ (see Fig. 1), namely

$\phi_{\mathrm{c}}(x)=\phi_{\mathrm{s}}(x) * g(x)$.

Depending on the technology, the smoothing function represents the writing spot of a laser beam or the line spread function of a lithographic process. For simplicity, we assume a normalized Gaussian signal for the smoothing function, namely

$g(x)=\frac{\sqrt{2 / \pi}}{w} \exp \left(\frac{-2 x^{2}}{w^{2}}\right)$,

where $x$ is the distance from the centre and $w$ is the half-width of $g(x)$ at $1 / \mathrm{e}^{2}$. The Gaussian smoothing is a model. It assumes a linear development characteristic which can be well achieved in the case of laser-writing [2]. Depending on the fabrication process, the model can be refined in order to include nonlinearities.
The smoothing procedure is presented in Fig. 1. In the upper line, the maximum phase change is $2 \pi$ ( $2 \pi$ design) and in the lower line the maximum phase change is $4 \pi$ ( $4 \pi$ design). In both cases, the number of phase levels per $2 \pi$ phase shift is equal to 2 . In general, the gratings have a phase depth of $m 2 \pi$ and a grating period of $m \Lambda$, where $m=1,2,3, \ldots$ If such a grating is illuminated perpendicularly at wavelength $\lambda$, then it deflects the incident beam by an angle $\theta$, given by $\sin \theta=\lambda / \Lambda$. The diffraction efficiency $\eta$ can be obtained from the Fourier-transform of the transmission function $\exp \left[\mathrm{i} \phi_{\mathrm{c}}(x)\right]$ [3]. The numerical values for the diffraction efficiency $\eta$ versus $w / \Lambda$ are presented in Fig. 2. The solid lines represent the efficiencies of the gratings resulting from the convolution of $4 \pi$ and $8 \pi$ deep ideally blazed gratings with the smoothing function. The dashed lines are the corresponding results for $4 \pi$ and $8 \pi$ deep multilevel gratings with 2 phase levels per $2 \pi$ phase change. For the discrete multilevel gratings $(w / \Lambda=0)$, the efficiency is for both gratings the same as for a binary grating, i.e. $40.5 \%$. If we now smooth the grating profile by increasing $w / \Lambda$, the efficiency increases also. For the 4-level design and a phase depth of $4 \pi$, the highest efficiency of $57.2 \%$ is reached at $w / \Lambda=0.207$. A still higher efficiency of $72.8 \%$ at $w / \Lambda=0.246$ can be achieved with same resolution for an 8-level design with phase depth of $8 \pi$. When the number of phase levels is doubled, i.e. 4 -levels per $2 \pi$ phase chage, the multilevel efficiency, $80.1 \%$, is again the same for $4 \pi$ and $8 \pi$ designs. The efficiencies of smoothed gratings of this type are presented in Fig. 2 by dash-dotted lines. The improvements in efficiency are now much 


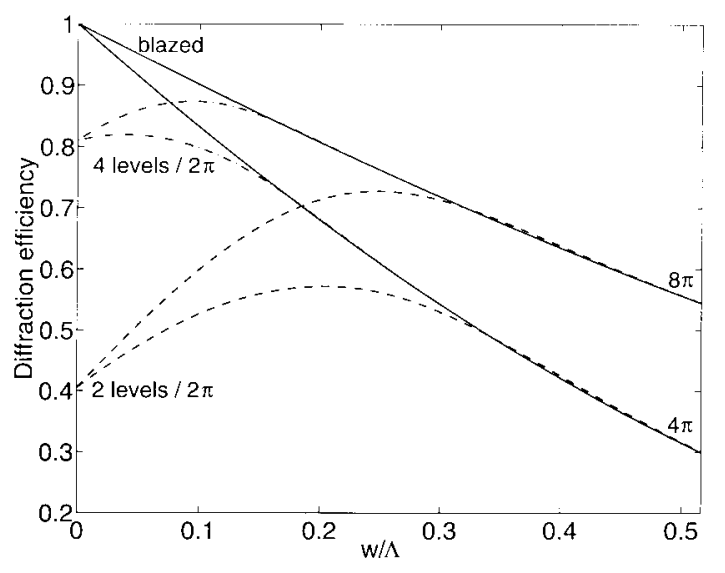

Fig. 2. Diffraction efficiencies of gratings convoluted with a Gaussian smoothing function versus $w / A$. The solid lines represent the efficiencies of the ideal blazed gratings after convolution. The efficiencies of the gratings with 2 phase levels per $2 \pi$ phase depth are presented by the dashed lines and the gratings with 4 phase levels per $2 \pi$ phase depth by the dash-dotted lines. For all line types the lower one corresponds to the $4 \pi$ phase depth design and the upper one to the $8 \pi$ phase depth design. Results are based on scalar theory.

smaller. For a phase depth of $4 \pi$ ( 8 -levels) no practical improvement is observed compared to the multilevel case. However, for a phase depth of $8 \pi(16-$ level) the highest efficiency is increased from $81 \%$ to $87.4 \%$ for $w / \Lambda=0.091$.

The convolution with a smoothing function introduces also losses because the phase transition of $m 2 \pi$ at the edges of the blazed gratings cannot be fabricated properly (see Fig. 1). For increasing width of the smoothing function, the losses become dominant and the efficiencies of the blazed grating and the multilevel approximation after convolution becomes equal. The losses depend on the ratio $d / m \Lambda$, where $d$ is the width of the phase transition region. The width $\mathrm{d}$ depends on the smoothing function $g(x)$ and does not change strongly with the relief height. Therefore the losses are smaller for deep structures $(m=2,3)$. Such gratings can be realised also in practice. Fabrication of diffractive components working in transmission with a phase depth of $8 \pi$ at $\lambda=632.8 \mathrm{~nm}$ and a local grating period of $5 \mu \mathrm{m}$ has been reported [2].

The multilevel grating structure and the smooth structures with $w / \Lambda=0.246$ (dashed-dotted line) and $w / \Lambda=0.516$ (dotted line) are shown in Fig. 3. Note that in the case of the highest efficiency at $w / \Lambda=$

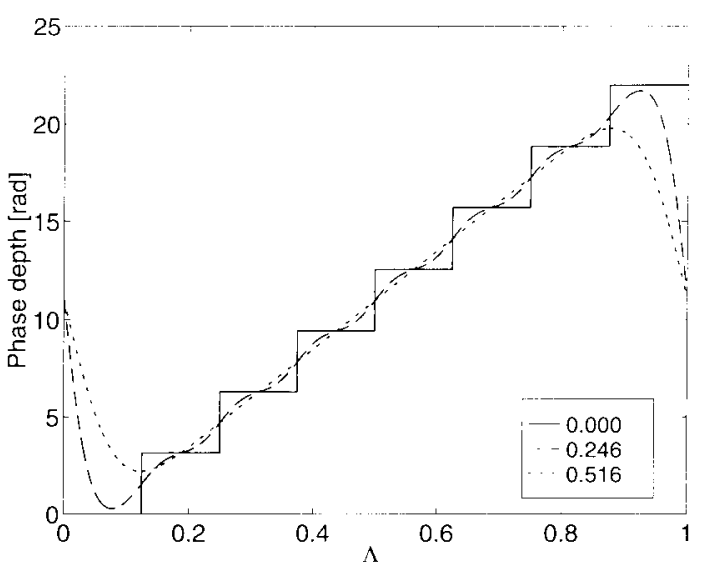

Fig. 3. Multilevel phase structure with maximum phase shift $8 \pi$ (continuous line) and the corresponding structure after smoothing (dashed line: $w / A=0.246$ and dotted line: $w / \Lambda=0.516$ ).

0.246 , the the phase excursion within one segment is the same as for the multilevel structure. Furthermore, the relief is still fluctuating. The fluctuation can be reduced by increasing $w / \Lambda$, but then the phase change is reduced also and with it the diffraction efficiency.

We have considered the validity of scalar theory by calculating the diffraction efficiencies of continuous grating structures rigorously. As a rigorous model we have used Knop's model [4] as described in Refs. [57 ]. The results for a $4 \pi$ design ( 2 phase levels per $2 \pi$ phase change) as a function of $w / \Lambda$ are presented in Fig. 4. The grating period $\Lambda$ is 10 times larger than the wavelength $\lambda$. The refraction indexes are $n_{1}=1$ for air and $n_{2}=n_{3}=1.5$ for grating material and substrate, respectively. The continuous line corresponds to the efficiency calculated by scalar theory ( same as the lower dashed line in Fig. 2), the dashed line represents TE-polarisation, and the dash-dotted line TMpolarisation. The stars are average values of these two polarisations. In this case rigorously calculated efficiencies behaves as predicted by scalar theory.

The highest efficiency for $4 \pi$ deep grating with 2 phase levels per $2 \pi$ phase shift is achieved around $w / \Lambda=0.222$. For this smoothed grating we calculated the diffraction behaviour as a function of $\Lambda / \lambda$. Because for small $\Lambda / \lambda$ ratios the diffraction efficiency depends on the propagation direction, we calculated both cases: the propagation from air through the grating to the substrate and the propagation from substrate through the grating to the air, see Fig. 5. The con- 


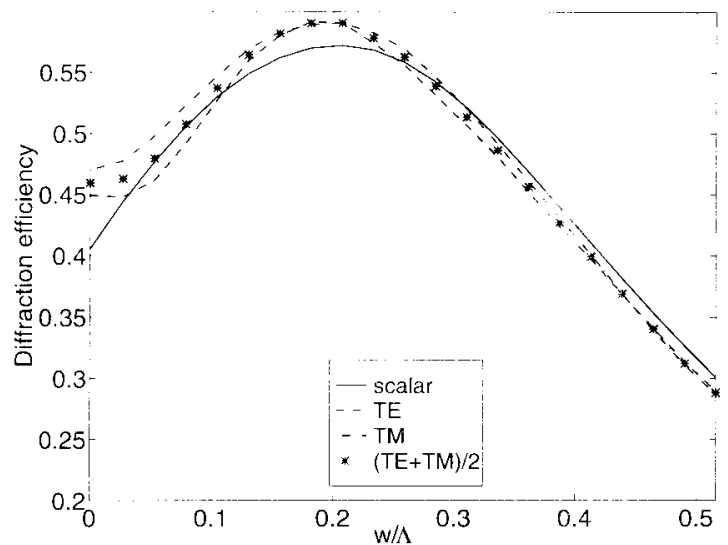

Fig. 4. Rigorously calculated diffraction efficiencies as a function of the smoothing factor $w / A$. The grating period $A$ is equal to 10 times the wavelength $\lambda(A / \lambda=10)$ and the refraction indices are $n_{1}=1$ and $n_{2}=n_{3}=1.5$. Continuous line: scaiar theory, dashed line: TE-polarisation, dash-dotted line: TM-polarisation, *: average values of TE- and TM-polarisation.

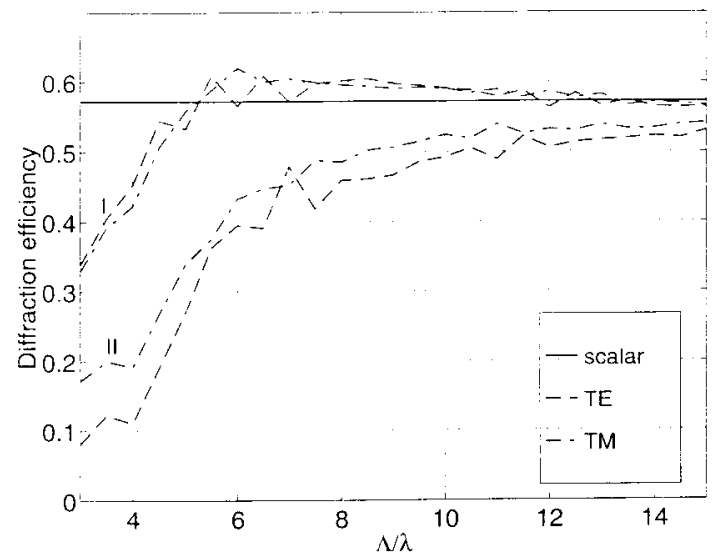

Fig. 5. Rigorously calculated diffraction efficiencies as a function of $\lambda / \lambda$ for two propagation directions: (I) from air to substrate and (II) from substrate to air. Continuous line: scalar theory, dashed line: TE-polarisation, dash-dotted line: TM-polarisation.

tinuous line represents the value obtained by scalar theory (independent of $\lambda / \lambda$-ratio), the efficiency for TE-polarisation is represented by the dashed line and the efficiency for TM-polarisation is represented by the dash-dotted line. In the direction air to substrate, the diffraction efficiencies reach the scalar value at $\Lambda / \lambda>5$ and the average efficiency is slightly above the scalar value when $A / \lambda$ is between 6 and 13. In the other propagation direction the $\Lambda / \lambda$ ratio have to be larger in order to achieve high efficiency. For large $A / \lambda$ ratios efficiencies for both propagation directions and for both polarisation converges to same value, which is a few percent lower than the value given by scalar theory. The difference is due to reflection losses at the interfaces.

\section{Continuous relief microlenses}

The grating period of diffractive microlenses is locally varying. Near the centre the grating periods are large and become then smaller towards the border. Thus, the central part could well be fabricated using multilevel technology. The improvements, discussed in the previous section, are interesting in the outer zone, where only 2 to 4 phase levels are realisable. The limit for a radius $r$, after which the lens structure becomes binary, is obtained from

$r=f \tan \left[\sin ^{-1}\left(\frac{\lambda}{3 \mathrm{mfs}}\right)\right]$,

where $f$ is the focal length of the lens and $\mathrm{mfs}$ is the minimum feature size which can be fabricated. For example, for a lens with a diameter of $1 \mathrm{~mm}$, a focal length of $f=1.5 \mathrm{~mm}$ at $\lambda=632.8 \mathrm{~nm}$ and $\mathrm{mfs}=$ $1 \mu \mathrm{m}$, this radius becomes $r=0.32 \mathrm{~mm}$. This value means that $59 \%$ of the lens area has a binary structure with $40.5 \%$ efficiency. If the efficiency can be improved in this region, it has strong effect on the total efficiency of the lens. In the following, we will consider microlenses, which have a large binary area at the border when the maximum phase change is assumed to be $2 \pi$. We will calculate the influence of smoothing on the efficiency of this kind of microlenses, when they are redesigned with a higher maximum phase change $(4 \pi$ or $8 \pi)$.

For lenses with small $F / \#$, we have used the Rayleigh-Sommerfeld diffraction integral to calculate the propagation of light from the element to the focal plane [8]. We have calculated the efficiency of the lens by integrating the intensity in the focal plane over a region which is three times the size of the diffraction limited spot. The results are then divided by the value of the integral, which is achieved with a highly sampled (ideal) lens function over the same region. In order to demonstrate the influence of the relief smoothing on the diffraction efficiency of microlenses, we consider cylindrical and spherical lenses 
Table 1

Diffraction efficiencies in $\%$ of cylindrical and spherical lenses for three different designs $(2 \pi, 4 \pi$ and $8 \pi)$ convoluted with three different gaussian signal widths $w / \Lambda_{M}\left(F / 1.5\right.$ and $\left.\Lambda_{M}=2 \mu \mathrm{m}\right)$

\begin{tabular}{|c|c|c|c|c|c|c|}
\hline \multirow[b]{2}{*}{$w / \Lambda_{M}$} & \multicolumn{3}{|c|}{ cylindrical } & \multicolumn{3}{|c|}{ spherical } \\
\hline & $2 \pi$ & $4 \pi$ & $8 \pi$ & $2 \pi$ & $4 \pi$ & $8 \pi$ \\
\hline 0.000 & 74.2 & 74.2 & 74.2 & 64.2 & 64.2 & 64.2 \\
\hline 0.222 & 64.0 & 76.2 & 83.9 & 52.9 & 69.1 & 79.3 \\
\hline 0.445 & 48.3 & 64.9 & $\overline{77.5}$ & 33.8 & 54.2 & $\overline{70.7}$ \\
\hline
\end{tabular}

of $F / 1.5$ operating at the wavelength $\lambda=632.8 \mathrm{~nm}$. For a $2 \pi$ realisation, this corresponds to a minimum period $\Lambda_{\mathrm{M}}$ of about $2 \mu \mathrm{m}$ at the border of the lens. Consequently, not more than 2 phase levels can be realised by using standard lithography. We calculated the diffraction efficiencies of three designs which have phase depths of $2 \pi, 4 \pi$ and $8 \pi$. In the centre, the lenses have 8 phase levels per $2 \pi$. Towards the border the number of levels decreases to 2 levels at minimum. The efficiencies for three different smoothing parameters $w / \Lambda_{M}$ are presented in Table 1. Note, that $\Lambda_{\mathrm{M}}$ is the minimum period for $2 \pi$ structures. For the multilevel structures $\left(w / \Lambda_{M}=0\right)$, the efficiencies for the cylindrical and spherical lenses are $74.2 \%$ and $64.2 \%$, respectively. In the case of a $2 \pi$ design the efficiency drops when the structure is smoothed, because smoothing leads to sinusoidal structures at the border of the lens (see also Fig. 1), which gives a lower efficiency than binary structures. In the case of a $4 \pi$ design with $w / \Lambda_{M}=0.222$, the efficiency is already higher than those achieved with the sampled phase structure $\phi_{s}(x)$. Significantly better results are obtained for the $8 \pi$ design, where the efficiency increases from $74.2 \%$ to $83.9 \%$ (cylindrical lens) and from $63.5 \%$ to $80.1 \%$ (spherical lens). We have also calculated the size of the focal spot according to the Rayleig-Sommerfeld diffraction integral. No significant increase of the focal spot diameter has been observed.

\section{Conclusions}

Blazed grating structures with a continuous profile have the highest efficiency. However, these ideal gratings cannot be fabricated in reality. Each technology has a finite resolution, which leads to losses.
We have compared binary technology with "smooth technology", such as gray-tone technology and direct writing. It turns out that for similarily sampled ideal phase function (only few samples per $2 \pi$ phase shift), the smooth profiles have higher diffraction efficiencies than the multilevel profiles if the phase modulation is $4 \pi$ and more. The improvements are significant for grating-like structures with periods in the order of a few microns, where only 2 or 4 phase levels per $2 \pi$ are feasible with standard binary optics technology.

We have also shown, that the typical criteria ( $\Lambda \sim$ $10 \lambda$ ) for validity of scalar theory holds also for these smoothed structures. However, we found a strong dependence on the propagation, with higher efficiency for the propagation from air to substrate. For that direction the scalar theory is already valid for $\Lambda / \lambda>5$, as indicated in Fig. 5.

\section{Acknowledgements}

M. Kuittinen acknowledges the Academy of Finland for financial support. Part of the work was supported by the Swiss Priority Program Optique.

\section{References}

[1] Y. Oppliger, P. Sixt, J.M. Stauffer, J.M. Mayor, P. Regnault and G. Voirin, One-step 3D shaping using gray-tone mask for optical and microelectronic applications, Microcircuit Engineering Conference 93, Maastricht, NL (1993).

[2] M.T. Gale, M. Rossi, J. Pedersen and H. Schütz, Opt. Eng. 33 (1994) 3556.

[3] J.W. Goodman, Introduction to Fourier Optics (McGraw-Hill, New York, 1968).

[4] K. Knop, J. Opt. Soc. Am. 68 (1978) 1206.

[5] D. Nyyssonen and C.P. Kirk, J. Opt. Soc. Am. A 5 (1988) 1270.

[6] E. Noponen and J. Turunen, J. Opt. Soc. Am. A 11 (1994) 1097.

[7] E. Noponen, Electromagnetic theory of diffractive optics, PhD. thesis, (Helsinki University of Technology, Dept. of Technical Physics, 1994).

[8] J.D. Gaskill, Linear Systems, Fourier Transforms, and Optics (Wiley, New York, 1978). 